\title{
Quantum Behavior Arises Because Our Universe is a Fractal
}

\author{
Yong Tao \\ College of Economics and Management \\ Southwest University, Chongqing, P. R. China \\ taoyingyong@yahoo.com \\ Received 11 February 2017 \\ Accepted 6 June 2017 \\ Published 6 July 2017 \\ Communicated by Ophir Flomenbom
}

\begin{abstract}
To explain the origin of quantum behavior, we propose a fractal calculus to describe the nonlocal property of the fractal curve [Y. Tao, J. Appl. Math. 2013 (2013) 308691]. This study demonstrates that if the dimension of time axis is slightly less than 1, then Planck's energy quantum formula will naturally emerge. In this paper, we further show that if the dimension of time axis is less than 1, Heisenberg's Principle of Uncertainty will emerge as well. Our finding implies that fractal calculus may be an intrinsic way of describing quantum behavior. To test our theory, we also provide an experimental proposal for measuring the dimension of time axis.

Keywords: Planck's constant; fractal calculus; Heisenberg's principle of uncertainty; quantum field theory; dimensional regularization.
\end{abstract}

PACS Numbers: 11.10.Kk, 11.10.Gh, 03.65.Ud, 98.80.-k

\section{Introduction}

The quantum field theory is one of the oldest fundamental and most widely used tools in physics. It is spectacularly successful in describing the behavior of microscopic particle. The quantum field theory is established on the basis of the theoretical frameworks including special relativity and quantum mechanics. The starting point of special relativity is the principle of invariant light speed, while the starting point of quantum mechanics is Planck's hypothesis of energy quantum, ${ }^{1,2}$ i.e.,

$$
\varepsilon=h \nu,
$$

where $h$ denotes the Planck's constant and $\nu$ the frequency.

It is well known that the principle of invariant light speed is an intrinsic requirement for the symmetry of Maxwell electromagnetism equations. Nevertheless,

This is an Open Access article published by World Scientific Publishing Company. It is distributed under the terms of the Creative Commons Attribution 4.0 (CC-BY) License. Further distribution of this work is permitted, provided the original work is properly cited. 
the hypothesis of energy quantum (1), which differs from the principle of invariant light speed, is a result of Max Planck's attempts to provide a theoretical explanation for the empirically discovered laws of blackbody radiation. Although the quantum mechanics provides a powerful way to our understanding of microscopic structure of matter, so far there is still no consensus on the origin of Planck's constant. For instance, it seems natural to ask whether the Planck's constant can be derived from a deeper principle.

Moreover, EPR paradox, presented by Einstein et al., ${ }^{3}$ indicates that the quantum entanglement would violate the principle of locality of field theory. For example, the locality of field might be violated within the Compton wavelength. ${ }^{4}$

In addition to violations of the principle of locality, there further exists ultraviolet divergence in quantum field theory, which needs to be removed by using the dimensional regularization method. ${ }^{5}$ This method requires that $\mathbf{S}$ matrix should be calculated in a fractional dimensional space-time..$^{5}$ Although nobody clarifies the physical meaning of fractional dimensional space-time, Wilson ${ }^{6}$ and Svozil $^{7}$ still developed the quantum field theory on fractal space-time (QFTFS), which further eliminates the "triviality" of $\lambda \phi^{4}$. In particular, Svozil's work implies that the dimension of space-time should be $D=4-\delta$, where $0<\delta \ll 1 .^{7}$ More importantly, recently, the investigation for quantum gravity strongly indicates that a power-counting renormalizable gravity model can be obtained in a fractional dimensional space-time. ${ }^{8-11}$

All of these developments for quantum field theory and quantum gravity above incarnate the popular notion that "the Universe is fractal" at quantum scales ${ }^{10-14}$ Although the notion of "fractal universe" is fascinating, there is no rigid calculus theory to describe the fractal. For example, Wilson ${ }^{6}$ and Svozil ${ }^{7}$ conjectured an integral formula for dealing with fractional dimensional manifold but left its rigid proof to mathematicians. Recently, we proposed a "fractal calculus" 15 by using fractional calculus, and employed it to verify the validity of Wilson and Svozil's conjectural integral formula. ${ }^{15}$ Using such a fractal calculus, we further show that ${ }^{16}$ if the dimension of time axis is slightly less than 1, then Planck's energy quantum formula (1) will naturally emerge. Based on our previous calculation, ${ }^{16}$ the dimension of time axis should approach $1-10^{-59}$, while the dimension of a space axis equals 1 . It coincides with Horava-Lifshitz gravity model ${ }^{8,9}$ which assumes that space and time are not equivalent (anisotropic) at high energy level (that is, the relativistic concept of time with its Lorentz invariance emerges at large distances). The main purpose of this paper is to show that if the dimension of time axis is less than 1 , then, by using fractal calculus, Heisenberg's Principle of Uncertainty will naturally emerge. Here we also provide an experimental proposal for measuring the dimension of time axis.

The organization of this paper is as follows. In Sec. 2, we introduce fractal calculus, and distinguish it from fractional calculus. In Sec. 3, we show that if the dimension of time axis is less than 1, then Heisenberg's Principle of Uncertainty will naturally emerge. In Sec. 4, we show that fractal calculus can be thought of as a possible mathematical basis for establishing quantum field theory on fractal space-time. In Sec. 5, we provide an experimental proposal for measuring the dimension of time axis. In Sec. 6, we present our conclusion. 


\section{Fractal Calculus}

In this section, we introduce fractal calculus. ${ }^{15}$ In classical mathematics, the dimension of a geometric graph is determined by the number of independent variables (i.e., the number of degrees of freedom). For example, every point on a plane can be represented by 2 -tuple real numbers $\left(x_{1}, x_{2}\right)$, then the dimension of the plane is denoted by 2 . Such a definition for dimension is intuitive. Unfortunately, mathematicians find many counter-examples. For instance, Peano's curve, which is determined by an independent characteristic parameter, would fill up the entire plane. ${ }^{17}$ Then, according to the classical definition of dimension, we have to conclude that the dimension of the plane equals 1 . This is a contradiction.

To avoid the dilemma of Peano's curve, mathematicians propose Hausdorff dimension, which later led to the development of fractal theory. ${ }^{18}$ Hausdorff dimension indicates that the dimension of a curve may take an arbitrary value (including fraction). In particular, if the dimension of a curve, $\omega$, is not equal to 1 , then it is called a $\omega$-dimensional fractal curve. From this meaning, Peano's curve is a 2-dimensional fractal curve. In general, the length of a $\omega$-dimensional fractal curve is denoted by

$$
|x(l)| \propto l^{\omega},
$$

where $l$ is an independent parameter which completely determines the generation of a $\omega$-dimensional fractal curve.

The length (2) can be obtained by evaluating the following Hausdorff measure:

$$
\lim _{\Delta l \rightarrow 0} \sum_{i}|x(l-i \Delta l)-x(l-(i+1) \Delta l)|^{\omega},
$$

where $x(l-i \Delta l)$ denotes a point on the $\omega$-dimensional fractal curve, and $i=0,1,2 \ldots{ }^{15}$

Although the Hausdorff measure is a useful tool to evaluate the length and dimension of a fractal curve, it fails to describe the non local property of the fractal, ${ }^{15}$ e.g. the self-similarity between local and global shapes of a fractal curve. To see this, we only need to note that, by using Hausdorff measure, the distance between $x(l)$ and $x(l-\Delta l)$ is denoted by $|x(l)-x(l-\Delta l)|^{\omega}$, which does not depend on $x(l-j \Delta l)$, where $j=2,3,4 \ldots{ }^{15}$ This contradicts the self-similarity of a fractal on which all the points will be influenced by each other.

To overcome the shortcomings of the Hausdorff measure, we propose to use the differences of order $\omega$ to evaluate the length of a $\omega$-dimensional fractal curve. ${ }^{15}$ Then the distance between $x(l)$ and $x(l-\Delta l)$ is defined in the form:

$$
\left|\Delta_{\omega}[x(l), x(l-\Delta l)]\right|=\left|\sum_{j=0}^{\infty} \frac{\omega(\omega-1) \cdots(\omega-j+1)(-1)^{j}}{j !} x(l-j \Delta l)\right|,
$$

where $\Delta_{\omega}$ denotes the difference operator of order $\omega$.

The measure (4) is called the "non-local distance". ${ }^{15}$ It implies that the distance between $x(l)$ and $x(l-\Delta l)$ will be influenced by all other points $x(l-j \Delta l)$, where 
$j=2,3,4 \ldots{ }^{15}$ This is a clear non-local correlation. Moreover, it is easy to check that $\left|\Delta_{\omega=1}[x(l), x(l-\Delta l)]\right|=|x(l)-x(l-\Delta l)|$. Thus, Euclidean distance is a special case of non-local distance (4).

As is well known, Newton-Leibniz calculus is based on Euclidean distance: The derivative of any function $y=f(x)$ is defined in the form:

$$
\frac{d f(x)}{d x}=\lim _{\Delta x \rightarrow 0} \frac{f(x)-f(x-\Delta x)}{\Delta x},
$$

where $|\Delta x|$ and $|f(x)-f(x-\Delta x)|$ denote Euclidean distances.

In the picture of Newton-Leibniz calculus, the Cartesian coordinates $(x, y)$ consist of two 1-dimensional lines (or curves). This is why the derivative (5) is based on Euclidean distance.

However, if the Cartesian coordinates $(x, y)$ consist of two $\omega$-dimensional fractal curves, then the derivative (5) breaks down. For the case of fractal coordinates, we propose to use the non local distance (4) to define the fractal derivative as below ${ }^{15}$ :

$$
\frac{{ }_{l} D_{\omega} f(x)}{{ }_{l} D_{\omega} x}=\lim _{\Delta l \rightarrow 0} \frac{\Delta_{\omega}[\bar{f}(l)-\bar{f}(l-\Delta l)]}{\Delta_{\omega}[x(l)-x(l-\Delta l)]} .
$$

where $\bar{f}(l)=f[x(l)]$ is a differentiable function and $l$ is an independent parameter which completely determines the generation of a $\omega$-dimensional fractal curve.

By using Riemann-Liouville fractional derivative, ${ }^{19}$ the fractal derivative (6) can be written in the form ${ }^{15}$ :

$$
\frac{{ }_{l} D_{\omega} f(x)}{{ }_{l} D_{\omega} x}=\frac{\frac{d^{\omega} \bar{f}(l)}{d l^{\omega}}}{\frac{d^{\omega} x(l)}{d l \omega}} .
$$

Equation (7) shows that fractal derivative is a ratio of two Riemann-Liouville fractional derivatives (rather than Caputo fractional derivative ${ }^{19}$ ). This is a key difference between fractional derivative and fractal derivative. By using the fractal derivative (6) or (7), we have shown that ${ }^{16}$ if the dimension of time axis is slightly less than 1 , one can derive the energy quantum formula (1). Before discussing this point, let us show how to construct a $\omega$-dimensional fractal curve. It can help us to understand a $\omega$-dimensional time axis.

The procedure of generating a $\omega$-dimensional fractal curve has been shown in Fig. 1. For simplicity, let the length of the interval $\left[y_{2}^{(1)}, y_{1}^{(1)}\right]$ equal 1 (see Fig. 1). It is easy to see that the Euclidean length of the interval $\left[y_{2}^{(n)}, y_{1}^{(n)}\right]$ equals $\left(\frac{1}{2}-\frac{1}{2 m}\right)^{n-1}$, and that the total number of remaining intervals equals $2^{n-1}$. Therefore, the Hausdorff dimension of this fractal curve equals

$$
\omega(m)=-\frac{\ln 2^{n-1}}{\ln \left(\frac{1}{2}-\frac{1}{2 m}\right)^{n-1}}=\frac{\ln 2}{\ln 2+\ln \frac{m}{m-1}} .
$$

Clearly, $\lim _{m \rightarrow \infty} \omega(m)=1$ and $\omega(m)$ is monotonically increasing with respect to $m$. This means that when $\omega(m)$ tends to 1 , fractal curve approaches continuum. Conversely, 
(a)

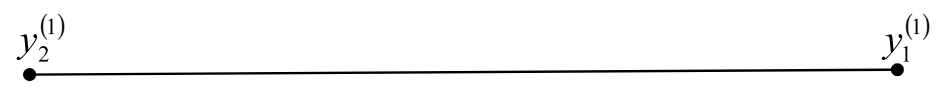

(b)

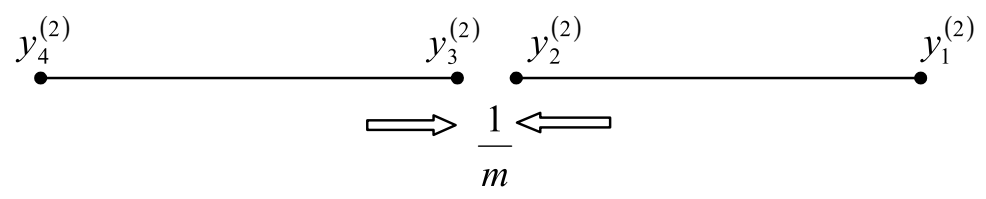

(c) $y_{2^{n}}^{(n)}$

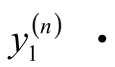

Fig. 1. The fractal curve with the dimension $\omega(m)=\frac{\ln 2}{\ln 2+\ln \frac{m}{m-1}}(m>1)$ is defined by repeatedly removing the middle $\frac{1}{m}$ of line segments: (a) One starts by removing the middle $\frac{1}{m}$ from the interval $\left[y_{2}^{(1)}, y_{1}^{(1)}\right]$, leaving $\left[y_{4}^{(2)}, y_{3}^{(2)}\right]$ and $\left[y_{2}^{(2)}, y_{1}^{(2)}\right]$. (b) Next, the "middle $\frac{1}{m}$ " of all remaining intervals is removed. (c) This process is continued ad infinitum. Finally, the $\omega(m)$-dimensional fractal curve consists of all points in the interval $\left[y_{2}^{(1)}, y_{1}^{(1)}\right]$ that are not removed at any step in this infinite process.

when $\omega(m)$ tends to 0 , the fractal curve yields a set of discrete points. Thus, the fractal curve in Fig. 1 can be regarded as a simple picture of understanding a $\omega$-dimensional time axis. It strongly implies that when the dimension $\omega$ is less than 1 , the time axis is discrete.

Finally, in Fig. 1, we attempt to provide a heuristic proposal to prove Cantor's continuum hypothesis; that is, there is no set whose cardinality lies between countable cardinality $a$ and uncountable cardinality $c$.

It is easy to check that when $m=3$, the fractal curve in Fig. 1 is the well-known Cantor ternary set. We have known that Cantor ternary set is uncountable. If we can prove that the fractal curve with the dimension $\omega(m)=\frac{\ln 2}{\ln 2+\ln \frac{m}{m-1}}$ for $m>1$ is uncountable, then, by imposing an axiom that the cardinality of a set is always monotonically non-decreasing with respect to the dimension of this set, we can verify that there are only two cardinality in the interval $[0,1]$; that is, countable cardinality $a$ and uncountable cardinality $c$. There would be no cardinality lying between $a$ and $c$. Here we have considered a fact that $\omega(m=1)=0$ is the dimension of a countable set (i.e., a set of discrete points) and $\omega(m=\infty)=1$ is the dimension of an interval $[0,1]$.

\section{Energy Quantum and Heisenberg's Principle of Uncertainty}

In this section, we show that if the dimension of time axis is less than 1 , then Heisenberg's Principle of Uncertainty will naturally emerge. To this end, let us make the following two assumptions ${ }^{16}$ :

Assumption (i). The total energy of the universe $E(t)$ is independent of time variable $t$; that is, $E(t)=E=$ const.

Assumption (ii). The dimension of time axis $\omega$ is slightly less than 1 . 
We have shown that ${ }^{16}$ by using fractal derivative (7), Assumptions (i) and (ii) will lead to the energy quantum formula (1). Let us first show it.

Assumption (ii) indicates that the time axis is a $\omega$-dimensional fractal curve, so its length yields $t \sim l^{\omega}$. Thus, by using formula (7), the fractal derivative of $E(t)$ with respect to the time axis $t$ follows $^{16}$ (or see the formula (B.1) in Ref. 15):

$$
\frac{{ }_{l} D_{\omega} E}{{ }_{l} D_{\omega} t}=\frac{E}{\Gamma(1-\omega) \Gamma(1+\omega)} \cdot \frac{1}{t},
$$

where $\Gamma(x)$ denotes the Gamma function.

Assumption (ii) indicates $1-\omega \rightarrow 0$, so Eq. (8) can be rewritten as ${ }^{16}$

$$
{ }_{l} D_{\omega} E=E \cdot(1-\omega) \cdot \frac{1}{t} \cdot{ }_{l} D_{\omega} t,
$$

where we have used $\lim _{\omega \rightarrow 1} \frac{1}{\Gamma(1-\omega) \Gamma(1+\omega)}=1-\omega$.

Let us order:

$$
\begin{gathered}
h=E \cdot(1-\omega) \cdot \Delta T, \\
\nu=\frac{1}{t}, \\
\Delta T={ }_{l} D_{\omega} t, \\
\varepsilon={ }_{l} D_{\omega} E,
\end{gathered}
$$

where $\Delta T$ denotes the critical time scale which can be regarded as a natural cutoff of the renormalization theory.

Substituting Eqs. (10)-(13) into Eq. (9), we obtain the energy quantum formula (1). Next we show that combining Eq. (10) and Assumption (ii) will lead to Heisenberg's Principle of Uncertainty.

Assumption (ii) indicates $0<\omega<1$, so we have:

$$
E \cdot \Delta T>E \cdot(1-\omega) \cdot \Delta T \text {. }
$$

Substituting Eq. (10) into Eq. (14) yields:

$$
E \cdot \Delta T>h
$$

Let us order

$$
\begin{gathered}
\Delta \varepsilon=\frac{E}{n}, \\
\Delta t=n \cdot \Delta T,
\end{gathered}
$$

where $n \geq 1$. By preceding discussion, $E$ indicates maximum energy scale and $\Delta T$ indicates minimum time interval, so $\Delta t$ and $\Delta \varepsilon$ denote the permitted time interval and energy scale, respectively; that is, $\Delta t \geq \Delta T$ and $\Delta \varepsilon \leq E$.

Substituting Eqs. (16) and (17) into Eq. (15), we obtain Heisenberg's Principle of Uncertainty:

$$
\Delta \varepsilon \cdot \Delta t>h .
$$


The process of deriving inequality (18) implies that quantum behavior may be related to fractal space-time. It is worth mentioning that other scholars also note this point. For example, Naschie, ${ }^{13}$ Adda and Porchon ${ }^{20}$ employ the nowhere differentiable function to derive Heisenberg's Principle of Uncertainty; Ord et al. ${ }^{21,22}$ employed random path (fractal path) to derive Schrodinger equation; Nottale et $a l .{ }^{23,24}$ developed the "Scale Relativity" to derive the postulates of quantum mechanics. However, our method differs from these excellent attempts. In contrast, we employ the fractal derivative (7) to derive Planck's energy quantum formula and Heisenberg's Principle of Uncertainty. Moreover, Assumption (ii) implies that the dimension of space-time is less than 4 . This is still not a new idea. In fact, many scholars have paid attention to it. ${ }^{5-7,25}$ In particular, by using quantum electrodynamics, Svozil has proposed a method for measuring the dimension of space-time. ${ }^{7}$ Different from Svozil's proposal, we directly employ the fractal theory to present an experimental scheme of measuring the dimension of time axis. ${ }^{16}$

\section{Quantum Field Theory on Fractal Space-Time}

Inequality (18) implies that fractal calculus may be an intrinsic way of describing quantum behavior. In fact, a consistent quantum theory should be established in a fractal space-time. This is why Wilson and Svozil developed QFTFS. ${ }^{6,7}$ Later, we will see that fractal calculus ${ }^{15}$ naturally leads to the theoretical framework of QFTFS.

In Sec. 3, we have shown that if the dimension of time axis is less than 1, one can obtain the energy quantum formula (1). This means that quantum behavior arises because the dimension of space-time, $D$, is less than 4 . By using available data, we have estimated ${ }^{16} D \sim 4-10^{-59}$ and $\omega \sim 1-10^{-59}$. Since $\omega$ approaches 1 so near, we cannot experience quantum phenomena in a macroscopic scale. However, quantum behavior will emerge in a microscopic scale, where space-time exhibits a pattern of fractal. Because the dimension of our space-time approaches 4 so near, NewtonLeibniz calculus will work very fine. This means that quantum theory based on Newton-Leibniz calculus almost always works well, except the extreme case where the fractal structure of space-time will play a non-negligible role, e.g., the critical time scale (so-called the cutoff of the renormalization theory) is involved.

By de Broglie's method, the energy quantum formula (1) will inevitably lead to matter wave $\phi(x, t)$. Thus, we can directly write down the path integral of any field $\phi(x, t)$ as

$$
Z=\int D \phi e^{\frac{i}{\hbar} \int d^{D} x \cdot \mathcal{L}\left(\phi, \partial_{\mu} \phi\right)},
$$

where $\mathcal{L}\left(\phi, \partial_{\mu} \phi\right)$ denotes the Lagrangian function of the field $\phi(x, t)$.

The starting point of QFTFS is to assume that the dimension of space-time, $D$, is less than 4. To guarantee that the path integral (19) works well at $D<4$ 
(e.g., $D=3.999 \ldots$ ), Wilson ${ }^{6}$ and Svozil $^{7}$ conjectured that the integral of any spherically symmetric function $f(r)$ on a $D$-dimensional space-time $\Omega$ should yield

$$
\int f(r) d^{D} x=\frac{2 \pi^{\frac{D}{2}}}{\Gamma\left(\frac{D}{2}\right)} \int_{0}^{R} f(r) r^{D-1} d r .
$$

In the past, Hausdorff measure was regarded as an ideal tool for describing the fractional dimensional manifold. ${ }^{7}$ Unfortunately, Hausdorff measure does not lead to formula (20). ${ }^{15}$ Therefore, QFTFS cannot be established in the mathematical framework of Hausdorff measure. However, we have proved that ${ }^{15}$ the formula (20) holds if the fractal calculus is taken into account. Next, let us show it.

By using the fractal derivative (6), fractal integral is defined in the form ${ }^{15}$ :

$$
\int{ }_{l} D_{\omega} f(x)=\int_{\beta_{\omega}(l)} \frac{{ }_{l} D_{\omega} f(x)}{{ }_{l} D_{\omega} x}{ }_{l} D_{\omega} x
$$

where the integral domain is a $\omega$-dimensional fractal curve $\beta_{\omega}(l)$.

By using the integral definition (21), the validity of the formula (20) can be verified. To see this, let us consider four fractal curves $\beta_{D_{i}}\left(l_{i}\right)$ with the dimensions $D_{i}$, respectively, where $i=0,1,2,3$. If we denote by $\beta_{D_{0}}\left(l_{0}\right)$ the time axis and denote by $\beta_{D_{j}}\left(l_{j}\right)$ the space axes $(j=1,2,3)$, then the Cartesian product of $\beta_{D_{i}}\left(l_{i}\right)$ can produce a $D$-dimensional space-time $\Omega$, where $D=D_{0}+D_{1}+D_{2}+D_{3}$ and $\Omega=\beta_{D_{0}}\left(l_{0}\right) \otimes \beta_{D_{1}}\left(l_{1}\right) \otimes \beta_{D_{2}}\left(l_{2}\right) \otimes \beta_{D_{3}}\left(l_{3}\right)$. If the spherically symmetric function $f(l)$ is defined on $\Omega$, then by using fractal integral (21) one can prove the following formula ${ }^{15}$ :

$$
\int_{\beta_{D_{0}}\left(l_{0}\right)} \int_{\beta_{D_{1}}\left(l_{1}\right)} \int_{\beta_{D_{2}}\left(l_{2}\right)} \int_{\beta_{D_{3}}\left(l_{3}\right)} f(l) \prod_{i=0}^{3} l_{i} D_{D_{i}} x_{i}=\frac{2 \pi^{\frac{D}{2}}}{\Gamma\left(\frac{D}{2}\right)} \int_{0}^{R} f(l) l^{D-1} d l,
$$

where $l^{2}=l_{0}^{2}+l_{1}^{2}+l_{2}^{2}+l_{3}^{2}$.

Clearly, the formula (22) confirms the validity of the formula (20). Therefore, fractal calculus can be regarded as a possible mathematical basis of establishing QFTFS. By investigating the Schrödinger equation, we have further shown that ${ }^{26,27}$ local description is not a way of completely describing quantum physics. In contrast, fractal calculus is just a non-local theory. ${ }^{15}$ Based on the advantages above, we believe that fractal calculus has the potential to become a natural way of describing quantum behavior.

\section{Experimental Test}

In the theoretical framework of QFTFS based on fractal calculus, the formula (10) is a key prediction, which can be experimentally tested. ${ }^{16}$ Formula (10) shows that Planck's constant may be expressed in terms of three fundamental constants: critical time scale, dimension of time axis and total energy of universe. In the past, we have proposed the corresponding methods to measure these three constants. ${ }^{16}$ Therefore, it is interesting to compare the well-known value of Planck's constant with the 
potential theoretical value consisting of three fundamental constants. Recently, Wilkinson Microwave Anisotropy Probe (WMAP) and Planck 2015 have brought the crucial data for estimating the total mass of our universe. ${ }^{28-30}$ By these data, we can estimate $E \sim 10^{51} \mathrm{~kg} .{ }^{16}$ Therefore, our main task at present is to measure $\omega$ and $\Delta T$. In Ref. 16, we propose to employ atomic clocks with different step lengths to complete the measurement. Here we will improve the previous proposal.

In accordance with the proposal of Ref. 16, we still use the following three steps to measure the length of a time interval, $L$.

(a) One uses a standard clock to specify a time interval whose length is $L$.

(b) One finds another clock $i$ whose step length is $L / N_{i}$, where $N_{i}>1$.

(c) Let the standard clock and the clock $i$ go simultaneously. Once the standard clock arrives at $L$ from 0 , one immediately counts the number of steps, $M_{i}=M_{i}\left(N_{i}\right)$, that the clock $i$ has gone.

Since the dimension of the time axis equals $\omega$, then by Mandelbrot's fractal theory ${ }^{18}$ one has

$$
\lim _{N_{i \rightarrow \infty}}\left(\frac{L}{N_{i}}\right)^{\omega} \cdot M_{i}=L^{\omega} .
$$

Equation (23) illustrates that when one uses the clock $i$ to measure " $L$ ", then the value of " $L$ " equals $L \omega$, where $\lim _{N_{i \rightarrow \infty}} \omega=\frac{\ln M_{i}}{\ln N_{i}}$.

In an actual measurement, one can use the clocks with different step lengths to approach the limit $N_{i} \rightarrow \infty$, that is,

$$
N_{1}<N_{2}<\ldots<N_{i}<\ldots<N_{n} .
$$

Because there exists a critical time scale $\Delta T$, the actual step length of the clock $i$ cannot approach zero. This means that the smallest step length is restricted by

$$
\lim _{N_{i \rightarrow \infty}} \frac{L}{N_{i}}=\Delta T .
$$

Therefore, using Eq. (25), Eq. (23) can be rewritten in the form:

$$
\left[\frac{1}{2}\left(\frac{L}{N_{i}}+\Delta T\right)\right]^{\omega} \cdot M_{i}=L^{\omega},
$$

for $i=1,2, \ldots, n$.

Here we have used $\lim _{N_{i \rightarrow \infty}} \frac{1}{2}\left(\frac{L}{N_{i}}+\Delta T\right)=\Delta T$. Equation (26) is a main improvement for the previous result. ${ }^{16}$

Taking the logarithm on both sides of Eq. (26), one has

$$
\omega \cdot\left[\ln \frac{1}{2}+\ln \left(\frac{1}{N_{i}}+\delta\right)\right]=\ln \left(\frac{1}{M_{i}}\right),
$$

where, $\delta=\frac{\Delta T}{L}$. 
Because $\Delta T$ or $\delta$ lies far beyond the reach of present-day experiments, one has $\delta \ll \frac{1}{N_{i}}$ for any $i$; therefore, one has the following Taylor expansion:

$$
\ln \left(\frac{1}{N_{i}}+\delta\right) \approx \ln \left(\frac{1}{N_{i}}\right)+N_{i} \cdot \delta .
$$

Substituting Eq. (28) into Eq. (27) yields

$$
\ln \frac{M_{i}}{N_{i}}=-\omega \cdot \delta+\omega \cdot \frac{\ln 2 N_{i}}{N_{i}} .
$$

If we order $y_{i}=\ln \frac{M_{i}}{N_{i}}, x_{i}=\frac{\ln 2 N_{i}}{N_{i}}$, and $\beta=-\omega \cdot \delta$, then Eq. (29) can be written in the form:

$$
y_{i}=\beta+\omega \cdot x_{i} .
$$

Obviously, using the measured value $\left(M_{i}, N_{i}\right)$ one can compute $\left(x_{i}, y_{i}\right)$, where $i=1,2, \ldots, n$. Then by the least square method, we have the following estimated values:

$$
\begin{aligned}
& \omega=\frac{\sum_{i=1}^{n} x_{i} y_{i}-\left(\frac{1}{n}\right) \cdot\left(\sum_{i=1}^{n} x_{i}\right) \cdot\left(\sum_{i=1}^{n} y_{i}\right)}{\sum_{i=1}^{n} x_{i}^{2}-\left(\frac{1}{n}\right) \cdot\left(\sum_{i=1}^{n} x_{i}\right)^{2}} \\
& \beta=\left(\frac{1}{n}\right) \cdot\left(\sum_{i=1}^{n} y_{i}\right)-\left(\frac{\omega}{n}\right) \cdot\left(\sum_{i=1}^{n} x_{i}\right) .
\end{aligned}
$$

As long as $\omega$ and $\beta$ are found by the formulas $\delta=\frac{\Delta T}{L}$ and $\beta=-\omega \cdot \delta$, one can get the estimated value of $\Delta T$. Using the estimated values of $\Delta T, \omega$ and $E$, we can obtain the theoretical value of Planck's constant.

\section{Conclusion}

We have shown that if the dimension of time axis is slightly less than 1 , Planck's energy quantum formula and Heisenberg's Principle of Uncertainty will naturally emerge in the mathematical framework of fractal calculus. Furthermore, because fractal calculus, which is a non local theory, provides a mathematical basis for establishing quantum field theory on fractal space-time, it may be considered as an intrinsic way of describing quantum behavior. To test our theory, we also provide an experimental proposal for measuring the dimension of time axis and critical time scale. We hope that the researcher who may be interested in our theory could make an effort to measure these two physical parameters.

\section{Acknowledgment}

This project was supported by the Fundamental Research Funds for the Central Universities (Grant No. SWU1409444). 


\section{References}

1. M. Planck, Ann. Phys. 4 (1901) 553.

2. A. Einstein, Ann. Phys. 17 (1905) 132.

3. A. Einstein et al., Phys. Rev. 47 (1935) 777.

4. E. M. Henley and W. Thirring, Elementrary Quantum Field Theory (McGraw-Hill, 1962), Chapt 5.

5. G. 't Hooft and M. Veltman, Nucl. Phys. B. 44 (1972) 189.

6. K. G. Wilson, Phys. Rev. D. 7 (1973) 2911.

7. K. Svozil, J. Phys. A: Math. Gen. 20 (1987) 3861-3875.

8. P. Horava, Phys. Rev. D. 79 (2009) 084008.

9. P. Horava, Phys. Rev. Lett. 102 (2009) 161301.

10. G. Calcagni, J. High Energy Phys. 3 (2010) 120.

11. G. Calcagni, Phys. Rev. Lett. 104 (2010) 251301.

12. M. S. El Naschie, Chaos Solitons Fractals. 1 (1991) 485-487.

13. M. S. El Naschie, Chaos Solitons Fractals. 2 (1992) 437-439.

14. M. S. El Naschie, Chaos Solitons Fractals. 3 (1993) 89-98.

15. Y. Tao, J. Appl. Math. 2013 (2013) 308691.

16. Y. Tao, ScienceOpen Research, 2015, doi: 10.14293/S2199-1006.1.SOR-PHYS.A7OCK4.v1.

17. H. Sagan, Space-Filling Curves (Springer-Verlag, 1994).

18. B. Mandelbrot, Science, 156 (1967) 636-638.

19. V. E. Tarasov, Fractional Dynamics: Applications of Fractional Calculus to Dynamics of Particles, Field and Media (Higher Education Press, Beijing, China, 2010).

20. F. B. Adda and H. Porchon, Rep. Math. Phys. 72 (2013) 201.

21. G. N. Ord and J. A. Gualtieri, Phys. Rev. Lett. 89 (2002) 250403.

22. G. N. Ord, Ann. Phys. 324 (2009) 1211-1218.

23. L. Nottale and M. N. Célérier, J. Phys. A, Math. Theor. 40 (2007) 14471-14498.

24. L. Nottale, Chaos Solitons Fractals, 25 (2005) 797-803.

25. M. S. El Naschie, World J. Nucl. Sci. Technol 4 (2014) 216-221.

26. Y. Tao, Commun. Theor. Phys. 56 (2011) 648-654.

27. Y. Tao, Commun. Theor. Phys. 57 (2012) 343-347.

28. D. N. Spergel et al., Astrophys. J. Suppl. Ser. 170 (2007) 377.

29. T. Gehrels, arXiv:astro-ph/0701344 (2007).

30. Planck Collab. P. A. R. Ade et al., Astron. Astrophys. 594 (2016) A13. 